$$
\text { for }
$$

\title{
Emulsion-Templated, Magnetic, Hydrophilic-Oleophobic Composites for Controlled Water Removal
}

\author{
Xiaomin $\mathrm{Li}^{\mathrm{a}}$, Tao Zhang ${ }^{\mathrm{a}, \mathrm{b}^{*}}$, Jintao $\mathrm{Lu}^{\mathrm{a}}$, Zhiguang $\mathrm{Xu}^{\mathrm{c}}$, and Yan $\mathrm{Zhao}^{\mathrm{a}, *}$ \\ a College of Textile and Clothing Engineering, Soochow University, Suzhou 215123, \\ China. \\ b China National Textile and Apparel Council Key Laboratory of Natural Dyes, Soochow \\ University, Suzhou 215123, China \\ ${ }^{c}$ China-Australia Institute for Advanced Materials and Manufacturing, Jiaxing University, \\ Jiaxing 314001, China.
}

Corresponding authors:

zhang.tao@suda.edu.cn (T. Zhang)

yanzhao@suda.edu.cn (Y.Zhao) 


\section{EXPERIMENTAL}

Table S1 Recipes for the MP- $X-Y_{S}$

\begin{tabular}{|c|c|c|c|c|c|}
\hline & MP-10-10 & MP-20-5 & MP-20-10 & MP-20-15 & MP-30-10 \\
\hline \multicolumn{6}{|c|}{ External, aqueous phase, wt.\% } \\
\hline FeNP suspension* & 2.00 & 4.00 & 4.00 & 4.00 & 6.00 \\
\hline $\mathrm{H}_{2} \mathrm{O}$ & 14.80 & 12.80 & 12.80 & 12.80 & 10.80 \\
\hline F127 & 1.20 & 1.20 & 1.20 & 1.20 & 1.20 \\
\hline AAm & 2.80 & 2.80 & 2.80 & 2.80 & 2.80 \\
\hline DEAEMA & 1.80 & 1.80 & 1.80 & 1.80 & 1.80 \\
\hline MBAAm & 0.50 & 0.50 & 0.50 & 0.50 & 0.50 \\
\hline APS** & 0.30 & 0.30 & 0.30 & 0.30 & 0.30 \\
\hline TEMED** & 0.16 & 0.16 & 0.16 & 0.16 & 0.16 \\
\hline Total & 23.56 & 23.56 & 23.56 & 23.56 & 23.56 \\
\hline \multicolumn{6}{|c|}{ Internal, organic phase, wt.\% } \\
\hline PFOTS & 6.92 & 3.46 & 6.92 & 10.38 & 6.92 \\
\hline Toluene & 69.52 & 72.98 & 69.52 & 66.06 & 69.52 \\
\hline Total & 76.44 & 76.44 & 76.44 & 76.44 & 76.44 \\
\hline \multicolumn{6}{|c|}{ FeNP dispersion in continuous phase $(\%)$} \\
\hline & 8.5 & 17.1 & 17.1 & 17.1 & 25.6 \\
\hline \multicolumn{6}{|c|}{ PFOTS in dispersed phase $(\%)$} \\
\hline & 9.1 & 4.5 & 9.1 & 13.6 & 9.1 \\
\hline $\operatorname{Density}(\mathrm{g} / \mathrm{cc})$ & 0.22 & 0.21 & 0.21 & 0.22 & 0.21 \\
\hline
\end{tabular}

*FeNP content at $1.88 \%$.

** APS dissolved in water $(0.5 \mathrm{~mL})$ and TEMED was added after HIPE formation. 


\section{RESULTS AND DISCUSSION}

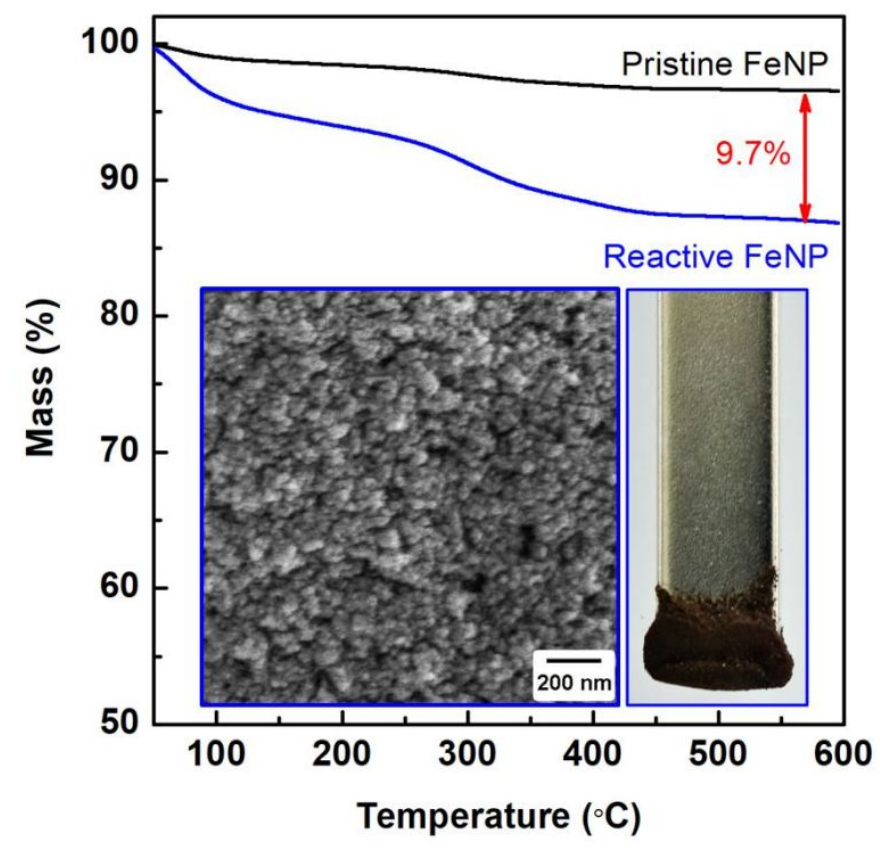

Figure S1. TGA curves of the pristine FeNP and reactive FeNP (the inserts showing typical morphology from SEM and a typical photo of FeNP absorbed by a magnetic bar).

From TGA curves in Figure S1, the pristine FeNP showed a negligible mass loss of 3.5\% after heating to $600{ }^{\circ} \mathrm{C}$, while the mass loss of the reactive FeNP increased to around $13.2 \%$. The relatively significant increase (around 9.7\%) in the mass loss could be ascribed to the loss of the organic component imposed by KH-570. The SEM micrograph inserted (Figure S1, insert) showed that the diameters of the reactive FeNP were around tens of nanometers, although the exact diameters were not able to determine. 


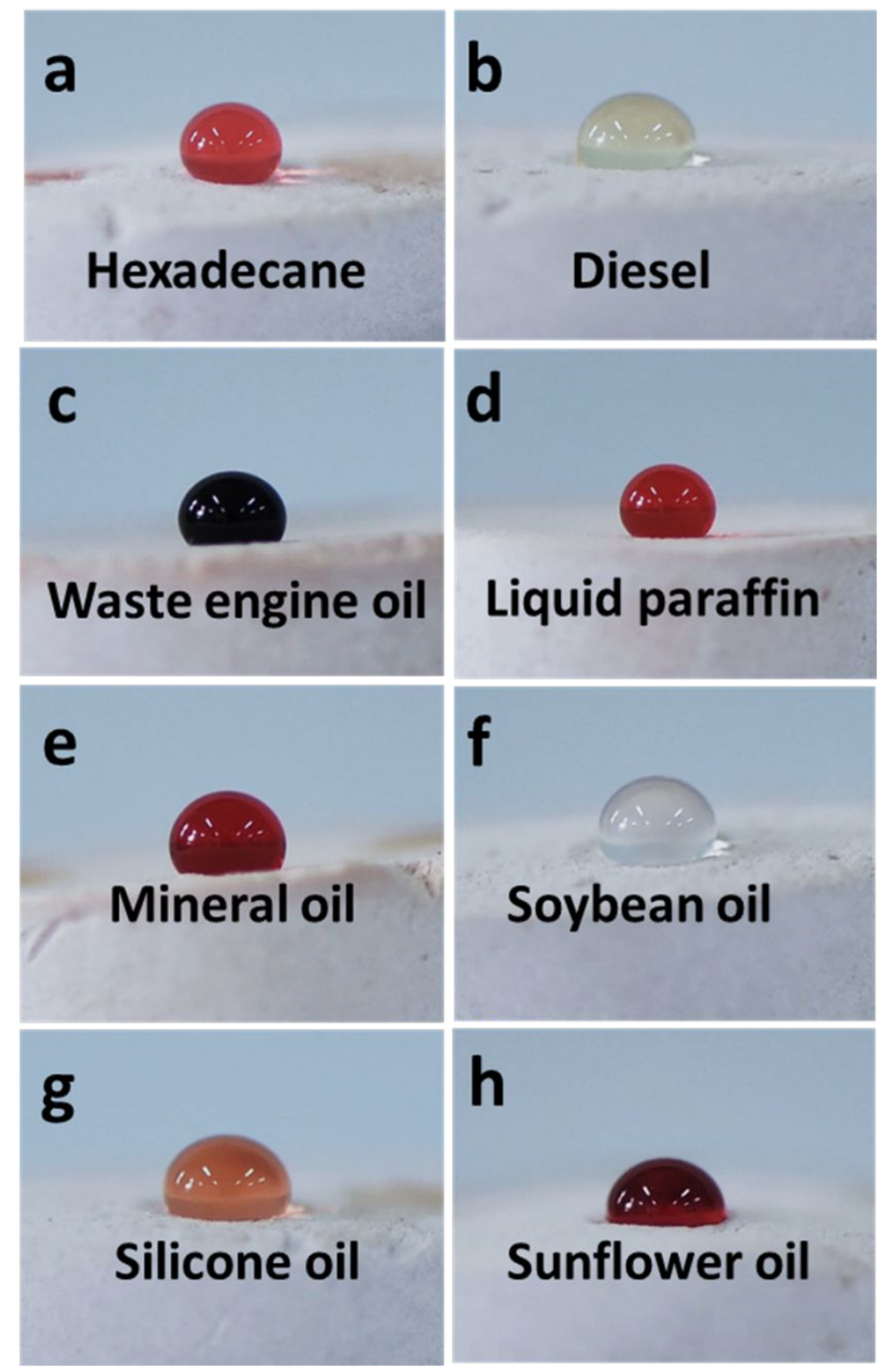

Figure S2. Photos of various oil droplets on MP-20-10. 

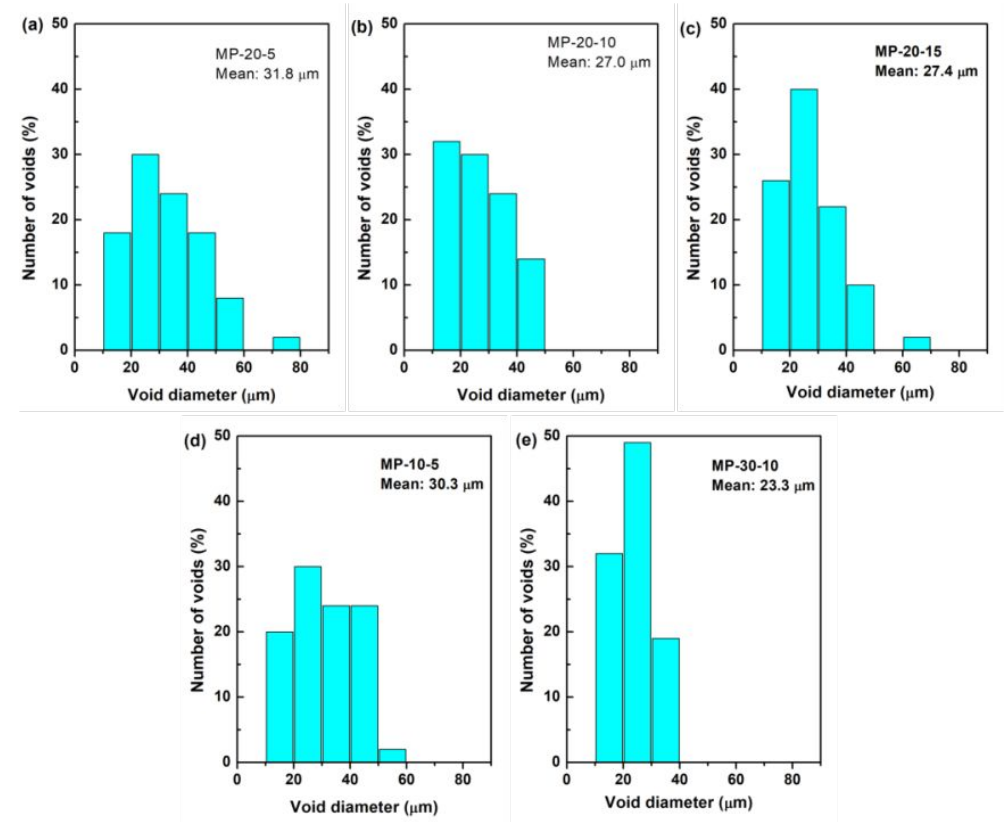

Figure S3. Void diameter distributions of the MP- $X-Y$ s: (a) MP-10-10; (b) MP-20-5; (c) MP-20-10; (d) MP-20-15; (e) MP-30-10.

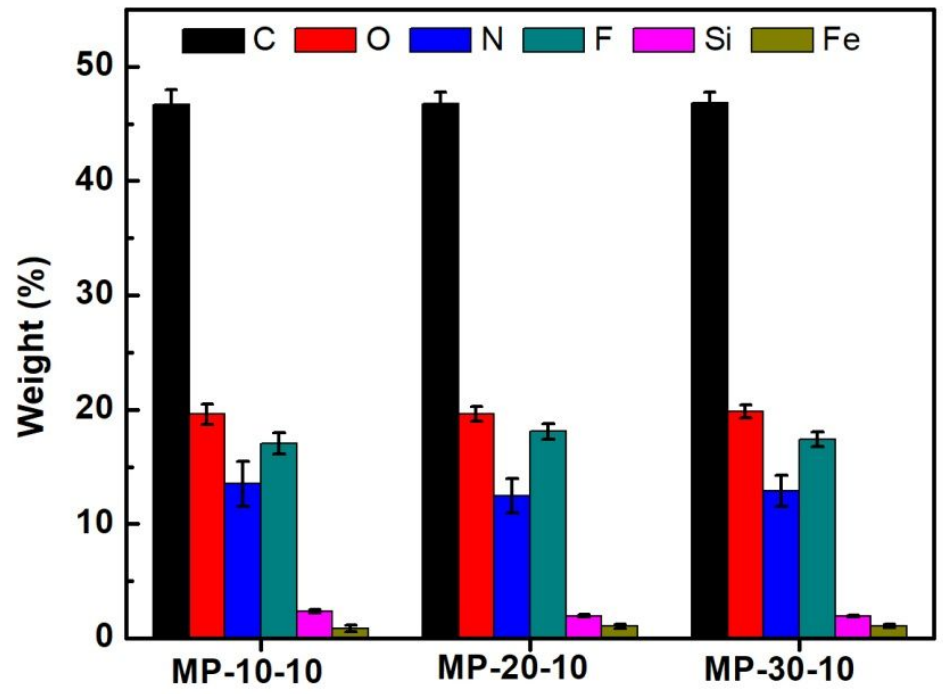

Figure S4. Elemental (C, N, O, F, Si and Fe) contents of the MP- $X-10$ determined by EDS 


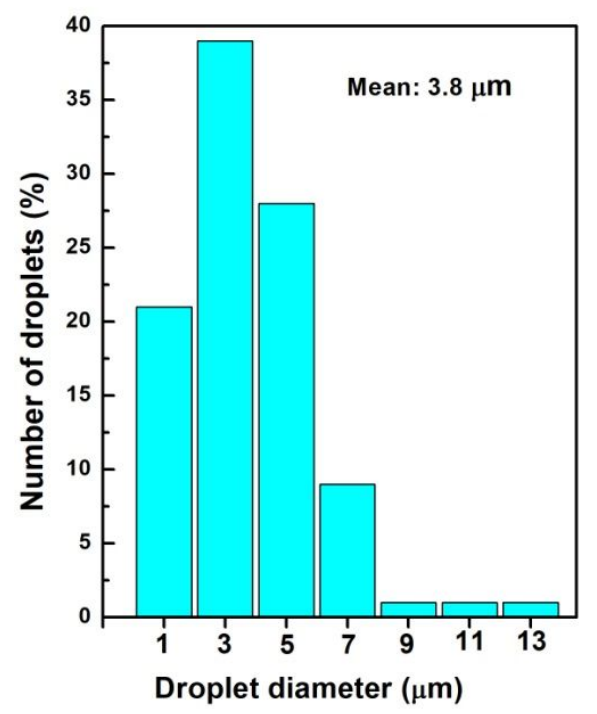

Figure S5. Dispersed droplet diameter distribution of water-in-diesel emulsion.

Video S1: Movement of dry MP-20-10 using a magnetic bar.

Video S2: Absorption of water droplets (dyed with methylene blue) under hexadecane using MP-20-10 and movement of water-swollen MP-20-10 using a magnetic bar. 